\title{
Stoppas Procedure: Place in present era
}

\author{
Dr. Lakshman Agarwal ${ }^{1}$, Dr. Sumita A Jain ${ }^{2}$, Dr. Arjun Rao Y S ${ }^{3}$, Dr. Prasant \\ Maskara ${ }^{4}$,Dr. Ashish Goyal ${ }^{5}$ \\ ${ }^{1}$ (Department of surgery, S M S Medical College/ Rajasthan University of health sciences, India) \\ ${ }^{2}$ (Department of surgery, S M S Medical College/ Rajasthan University of health sciences, India) \\ ${ }^{3}$ (Department of surgery, S M S Medical College/ Rajasthan University of health sciences, India) \\ ${ }^{4}$ (Department of surgery, S M S Medical College/ Rajasthan University of health sciences, India) \\ ${ }^{5}$ (Department of surgery, S M S Medical College/ Rajasthan University of health sciences, India)
}

\begin{abstract}
:
Background: Inguinal hernias are frequently encountered disease. When not treated may lead to their complications like obstruction and strangulation. Though there has been extensive search but no one has been able to find the ideal way to repair inguinal hernia.

Aim: To evaluate and analyze the effectivity of Stoppa procedure in the management of bilateral inguinal hernia.

Patients and methods: study was carried out between October 2011 and January 2013. Hundred patients of bilateral inguinal hernia were operated using Stoppa Technique. Of these 12 were cases of recurrent hernia. The parameters evaluated were operative time, length of hospitalization, minor complications, recurrence and chronic groin pain.

Results: The outcome at the end of 1 yr follow-up was good. Average duration of surgery was $38.5 \mathrm{~min}$. In the early post operative period 3 patients had seroma, 3 patients had spinal headache, and a single case of urinary retention. 93 patients were discharged on the day after operation. At the end of 2 year follow-up none had recurrence or chronic groin pain.

Conclusions: Stoppa procedure is an operation devised to cure hernias and also prevented other types of hernia occurring through myopecteneal orifice. Its low complication and recurrence rates have made it an attractive option for hernia therapy.
\end{abstract}

Keywords: Hernia repair, Stoppas,

\section{Introduction}

Hernia surgery has one of the longest of histories, which is far from complete. Many great men have been devising innovative methods to repair hernia surgically. First were the anatomical repairs amongst which bassini's and shouldice's repairs merit mention. Later the use of prosthesis came into practice. This drastically improved the rate of recurrences. With the acceptance of mesh usage, many pioneer surgeons came up with indigenous methods of using it. With the usage of mesh came the question of which plain the mesh is to be placed.

Stoppa procedure involves the placement of a large prosthetic material in the preperitoneal space deep to the transversalis fascia to cover the myopecteneal orifices on both sides. The mesh acts as an artificial endoabdominal fascia preventing the visceral sac extension through the defect. The increased intra abdominal pressure, the causative factor, presses the mesh against the abdominal wall, becoming a preventive factor for recurrence and herniation through other weaknesses.

Stoppa procedure, being a posterior preperitoneal procedure, involves dissection in a plane with no nerves and minimal cord handling, thus avoiding inguinodynia and testicular atrophy.

This procedure, being in a different plane as compared to the conventional Lichtenstein repair, is preferred when operating a case of its recurrence (so called virgin plane).

\section{Patients And Methods}

All patients admitted with bilateral inguinal hernias, between October 2011 and March 2012, were included in the study. Even patients with recurrence were included. Patients presenting with strangulated hernia, obstructed hernia, with a midline scar from a previous laparatomy and those suffering from hemorrhagic tendency were excluded.

These patients were operated under spinal anaesthesia. Preoperatively patients were catheterised. The lower midline incision was put and the subcutaneous tissue dissected. The anterior rectus sheath is incised in the midline. The underlying rectii are separated from each other to reach the preperitoneal space. Dissection progressed laterally under the rectus abdominis muscle and posteriorly to the inferior epigastric vessels. This 
cleavage is continued down-ward, anterior to the bladder, to the prostatic fossa, and behind the iliopubic ramus in the space of Bogros. The pedicle of the hernia is isolated, either distinct from the spermatic cord (in direct inguinal or femoral hernias) or connected to it (in indirect inguinal hernias). The preperitoneal cleavage is then continued below the external iliac vessels and laterally along the psoas muscle. Parietalization of the components of the spermatic cord is undertaken. A large mesh of width corresponding to two centimetres less than the distance between the two anterior superior iliac spines and vertical length corresponding to the distance between the umbilicus and the pubic symphysis is laid in the dissected preperitoneal space. Closure was done in layers. No drains were inserted. Operation time was noted.

Post operative course in the hospital was noted for pain and other early post operative complications.

Patients were evaluated on POD 10, 1 month, 6 months, 9 months and 12 months after surgery for minor complications, recurrence and chronic groin pain

\section{Results}

Hundred patients were included in the study. All were males. Age was between 40 and 72 years with a mean age of 57.52 years. Out of these patients, the types of hernia and sides involved are shown in Table 1. All patients underwent Stoppa repair without drainage procedure under spinal anaesthesia. Incision used was lower midline incision as all patients had bilateral hernias.

Average time taken during surgery was $38.5 \mathrm{~min}$, minimum time being $30 \mathrm{~min}$ and maximum being 48 $\min$.

Ninety three patients were discharged with in $24 \mathrm{hrs}$ of the procedure, after removal of catheter. Complications occurred in $5(5 \%)$ patients thus causing a delayed in discharge from hospital. Early recurrence occurred in none of the patients. Complications that occurred were urinary retention in $1(1 \%)$ patients and seroma occurred in 3 (3\%) patients, managed with aspiration. Superficial or deep wound infections occurred in none of the patients. Three (3\%) patient had spinal headache. Average hospital stay was 1.1 days.

On long term follow-up none of the patients had recurrence or chronic groin pain. There was no mortality.

\section{Conclusions}

Stoppa technique is an excellent technique. It provides a safe and definitive cure, especially in cases of bilateral and recurrent hernia. Because of the simplicity, excellent results and avoidance of chronic pain, it has become an effective add on to the surgeons' arsenal to treat bilateral, recurrent and complex (sliding) hernias.

\section{Figures and Tables}

\section{Table 1: Distribution of Hernia}

\begin{tabular}{|c|c|c|c|}
\hline & Direct & Indirect & Combined \\
\hline Right & 60 & 32 & 8 \\
\hline Left & 63 & 34 & 3 \\
\hline
\end{tabular}

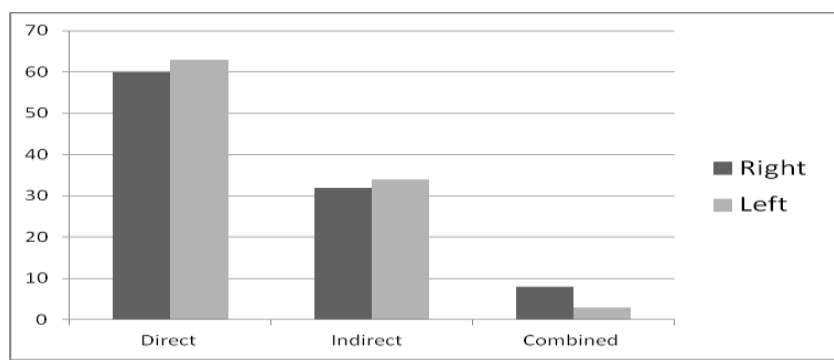

Figure 1: Distribution of Hernia

Table 2: Complications

\begin{tabular}{|l|l|l|}
\hline Complications & No. of patients & Percentage (in \%) \\
\hline Early & & \\
\hline Seroma & 3 & 3 \\
\hline Spinal headache & 3 & 3 \\
\hline Urinary retention & 1 & 1 \\
\hline Wound infection & 0 & 0 \\
\hline Early Recurrence & & \\
\hline Late & & \\
\hline Late Recurrence & 0 & 0 \\
\hline Chronic groin pain & 0 & 0 \\
\hline MORTALITY & 0 & 0 \\
\hline
\end{tabular}

